\title{
Humanoid Synthesis using Clifford Algebra
}

\author{
Maria Cruz Villa Uriol \\ Calit2 Center of GRAVITY \\ University of California, Irvine \\ Irvine, CA 92697, USA \\ mvillaur@uci.edu
}

\author{
Alba Perez Gracia \\ School of Engineering \\ Idaho State University \\ Pocatello, ID 83209, USA \\ perealba@isu.edu
}

\author{
Falko Kuester \\ Calit2 Center of GRAVITY \\ University of California, Irvine \\ Irvine, CA 92697, USA \\ fkuester@uci.edu
}

\begin{abstract}
One of the challenges in the simulation of human motion, either applied to humanoid robots or avatars in virtual environments, is to design a kinematics structure and a set of joint trajectories that move a robot or avatar in a human-like manner. In this paper, a technique is introduced to create accurate humanlike motion with a simplified topology as a reference. Using an optical motion capture system, a finite number of key poses are captured from different subjects performing full body articulated movements. Motion is modeled using the Clifford algebra of dual quaternions and dimensional synthesis techniques are applied to generate the kinematic skeleton of a 3D avatar or robot. The synthesized kinematic skeleton provides location of joints and dimensions of the links forming the limbs, as well as the joint trajectories. Five serial chains constitute our approximation to the human skeleton. Revolute, universal and spherical joints are employed, although other topologies can be used in a similar fashion. Several real datasets are evaluated and results demonstrate that good accuracy can be obtained at interactive rates using the presented methodology. The results show that using simple serial chains in combination with dimensional synthesis suffices to generate the mechanical structure and trajectories of a humanoid robot or 3D avatar mimicking human motion.
\end{abstract}

\section{INTRODUCTION}

A broad range of applications can benefit from access to libraries of realistic human motion, from virtual environments to computer animated movies. If human motion is compactly expressed using robotics techniques, it can be applied to humanoid robot motion, in path planning and robot design.

Different types of model acquisition, motion capture and synthesis systems have been developed. O'Brien et al. [1] used magnetic motion captured data to determine the joint parameters of articulated figures to support system calibration. Articulated models have been described as products of exponential maps and twist motions for video-based [2] and voxel-based human motion tracking [3]. Kinematic models of articulated figures in volume sequences were extracted using Isomap [4]. Usually these techniques rely on considering a model in which the limbs can freely rotate about the joints, generating a set of rotations for each joint. However, these motions may not be directly accomplished by a humanoid robot having more restricted mobility, for instance using only revolute joints.

To analyze how human motion can be applied to humanoid robots, a set of characteristics needs to be extracted before motion can be mapped to robots with dimensions different from those of the captured subject [5], [6]. Similarly, motion retargetting has to address this problem when animating synthetic characters [7], [8]. Physical skills acquisition, for which motion has to be carefully evaluated and correlated, has similar demands [9].

Our research is oriented towards generating the motion and a simplified kinematic structure that can describe it [10], [11]. Both are accomplished by expressing the human body as a set of five kinematic serial chains, called the kinematics skeleton, while tracking information is obtained from optical motion capture data. Clifford algebra of dual quaternions is used to represent the corresponding human avatars. Clifford algebra has been used for motion interpolation [12], in robot design [11], in camera calibration and 3D rigid motion estimation [13].

The kinematics equations of the serial chain are equated to the target motion and solved for the joint angles and dimensions along the chain [14]. Without user intervention, it is then possible to obtain (i) a compact representation of the properties of the target subject's kinematic skeleton and (ii) a set of control parameters representing the motion of the kinematic skeleton for further use in animation or trajectory planning, at interactive rates.

The Clifford algebra of dual quaternions is briefly presented (Sect. II) to define the kinematics equations representing an arbitrary kinematic chain and to perform the synthesis (Sect. III) from a set of finite positions. In Section IV, the topology of the kinematic skeleton is described along with the kinematic chains to be dimensioned. Next, the kinematics equations and design equations for each chain are derived, and its solution process specified. Finally, the results are detailed (Sect. V) and summarized (Sect. VI).

\section{AnAlysis OF KinEMATiC CHAins}

\section{A. Kinematics Equations of a Serial Chain}

For a serial chain, the kinematics equations define the position of its end-effector as a function of the geometry of the chain and the joint variables. The most common formulation uses the Denavit-Hartenberg parameters [15] to define transformations between local frames, see Figure 1.

For the $m$-jointed kinematic chain, the kinematics equations at instant of time $t_{i}, i=1, \ldots n$, are defined as $\left[D_{i}\right]$ and are created as the composition of local screw displacements, 


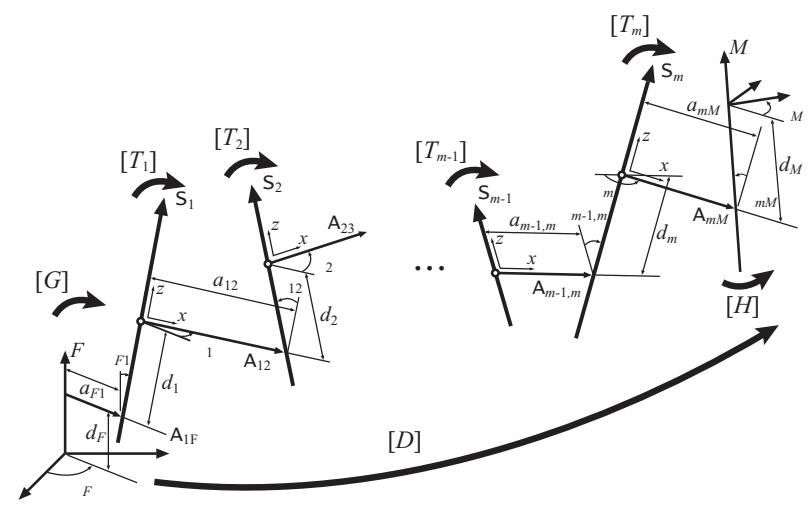

Fig. 1. Generic serial chain with $m$ joints.

expressed as homogeneous transformations,

$$
\begin{aligned}
{\left[D_{i}\right]=} & {[G]\left[Z\left(\theta_{1}^{i}, d_{1}^{i}\right)\right]\left[X\left(\alpha_{12}, a_{12}\right)\right]\left[Z\left(\theta_{2}^{i}, d_{2}^{i}\right)\right] \ldots } \\
& {\left[X\left(\alpha_{n-1, n}, a_{n-1, n}\right)\right]\left[Z\left(\theta_{m}^{i}, d_{m}^{i}\right)\right][H], \quad i=1, \ldots n, }
\end{aligned}
$$

where $[G]$ locates the base of the chain with respect to the fixed reference frame $F$, and $[H]$ represents the displacement between the end-effector and the last joint $m$.

Given $\left[D_{i}\right]$ at $t_{i}$ and $\left[D_{0}\right]$ at $t_{0}$, we can define the relative displacement $\left[D_{0 i}\right]=\left[D_{i}\right]\left[D_{0}\right]^{-1}$ which represents a displacement from the reference configuration $\left[D_{0}\right]$ expressed in the fixed frame. The kinematics equations of a relative displacement take the form

$$
\left[D_{i}\left(\Delta \overrightarrow{\theta^{i}}\right)\right]=\left[T\left(\Delta \theta_{1}^{i}, \mathrm{~S}_{1}\right)\right]\left[T\left(\Delta \theta_{2}^{i}, \mathrm{~S}_{2}\right)\right] \ldots\left[T\left(\Delta \theta_{m}^{i}, \mathrm{~S}_{m}\right)\right],
$$

where $S_{j}$ are the Plücker coordinates of the $j$ joint axis at the reference configuration expressed in the fixed frame, $\mathrm{S}_{j}=$ $\mathbf{S}_{j}+\epsilon \mathbf{S}_{j}^{0}$, and $\Delta \theta_{j}^{i}=\theta_{j}^{i}-\theta_{j}^{0}$ is the angle about joint $j$ measured from the reference configuration.

\section{B. Dual Quaternion Kinematics Equations}

The Clifford algebra of the projective three-space $\mathbb{P}^{3}$ is a sixteen-dimensional vector space with a non-commutative product called geometric or Clifford product [16], [17]. The elements of even rank form an eight-dimensional subalgebra $C^{+}\left(\mathbb{P}^{3}\right)$ that can be identified with the set of $4 \times 4$ homogeneous transformations. An element of $C^{+}\left(\mathbb{P}^{3}\right)$ can be written as the eight-dimensional vector given by

$$
\hat{S}=s_{0}+s_{1} i+s_{2} j+s_{3} k+s_{4} \epsilon+s_{5} i \epsilon+s_{6} j \epsilon+s_{7} k \epsilon,
$$

where $i, j$, and $k$ are the quaternion units, and $\epsilon$ is called the dual unit, with the property $\epsilon^{2}=0$.

In the calculations, the notation $\mathbf{S}=s_{1} i+s_{2} j+s_{3} k$ and $\mathbf{S}^{\circ}=s_{5} i+s_{6} j+s_{7} k$ is used allowing us to represent the Clifford algebra element (Eq. (2)) as

$$
\hat{S}=s_{0}+\mathbf{S}+s_{4} \epsilon+\mathbf{S}^{\circ} \epsilon=\left(s_{0}+s_{4} \epsilon\right)+\left(\mathbf{S}+\mathbf{S}^{\circ} \epsilon\right)=\hat{s}+\mathbf{S} .
$$

A spatial displacement is identified with the unit dual quaternion

$$
\hat{Q}=\cos \frac{\hat{\phi}}{2}+\sin \frac{\hat{\phi}}{2} \mathrm{~S},
$$

where $S$ is the screw axis of the displacement, and $\hat{\phi}$ is the dual axis variable defined as $\hat{\phi}=\phi+\epsilon t$, where $t$ is the translation along and $\phi$ is the rotation about the axis.

The composition of these Clifford algebra elements defines the relative kinematics equations for a serial chain that are equivalent to Eq. (1),

$$
\hat{D}_{i}(\Delta \hat{\vec{\theta}})=\hat{Q}_{1}\left(\hat{\theta}_{1}^{i}\right) \hat{Q}_{2}\left(\hat{\theta}_{2}^{i}\right) \ldots \hat{Q}_{m}\left(\hat{\theta}_{m}^{i}\right),
$$

where $\hat{Q}_{j}\left(\hat{\theta}_{j}^{i}\right)=\cos \frac{\Delta \hat{\theta}_{j}^{i}}{2}+\sin \frac{\Delta \hat{\theta}_{j}^{i}}{2} S_{j}$ corresponds to the relative transformation of the chain about joint $j$ from the reference configuration $\hat{D}_{0}$.

\section{Synthesis of Kinematic Chains}

\section{A. Design Equations}

Dimensional synthesis seeks to compute the dimensions of a kinematic chain in order to perform a specified task [16]. For our problem, the goal of the synthesis process is to determine the location and orientation of the human joints and the joint angles needed to perform a movement obtained via motion capture. For this, a series of $n+1$ coordinate data frames is given, from which we can calculate $n$ relative unit dual quaternions $\hat{P}^{i}, i=1 \ldots n$ expressing the relative motion of each link. First, the task dual quaternions $\hat{P}^{i}$ are equated with (Eq. (3)) to create the design equations $\mathcal{Q}_{i}$ for $i=1 \ldots n$

$$
\mathcal{Q}_{i}: \quad \hat{Q}_{1}\left(\hat{\theta}_{1}^{i}\right) \hat{Q}_{2}\left(\hat{\theta}_{2}^{i}\right) \ldots \hat{Q}_{m}\left(\hat{\theta}_{m}^{i}\right)-\hat{P}^{i}=0 .
$$

It is then possible to solve the set of $8 n$ design equations to obtain the location and orientation of the joint axes in the reference configuration $\hat{D}_{0}$. In addition, the inverse kinematics to reach the end-effector position can be obtained, that is, the relative joint angles $\theta_{j}^{i}$ for the task position $i$ at joint $j$.

\section{Kinematic Skeleton Synthesis}

\section{A. Topology and Joint Types}

Our human body model consists of five serial kinematic chains corresponding to the main limbs: head, arms and legs (Fig. 2). They are designed using spherical (S), universal (T) and revolute $(\mathrm{R})$ joints. An R-joint allows a one-degree-offreedom rotational movement, a T-joint allows a two-degreesof-freedom rotation expressed as two perpendicular R-joints concurrent at the joint location, while an S-joint allows a general rotation (three degrees of freedom) about a point. The skeleton to be designed has a total of 13 joints and 14 links, accounting for 27 degrees of freedom.

In Figure 2, an $\mathrm{S}$ joint is represented as a ball-joint, and the $\mathrm{R}$ and $\mathrm{T}$ joints are represented lines locating the rotation axes. Neck, shoulders and hips are modeled using $\mathrm{S}$ joints; elbows and knees as $\mathrm{T}$ joints; and wrists and ankles with $\mathrm{R}$ joints.

An R-joint is represented using dual quaternions as

$$
\hat{R}(\theta)=\left\{\begin{array}{c}
\sin \frac{\theta}{2} g_{x} \\
\sin \frac{\theta}{2} g_{y} \\
\sin \frac{\theta}{2} g_{z} \\
\cos \frac{\theta}{2}
\end{array}\right\}+\epsilon\left\{\begin{array}{c}
\sin \frac{\theta}{2} g_{x}^{0} \\
\sin \frac{\theta}{2} g_{y}^{0} \\
\sin \frac{\theta}{2} g_{z}^{0} \\
0
\end{array}\right\},
$$




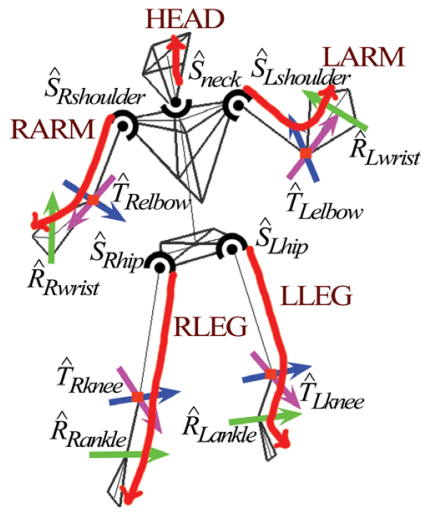

Fig. 2. Human body model.

where $\mathbf{g}=\left(g_{x}, g_{y}, g_{z}\right)$ is the rotation axis, $\mathbf{g}^{0}=\left(g_{x}^{0}, g_{y}^{0}, g_{z}^{0}\right)$ gives its location, and $\theta$ is the rotation angle about $\mathbf{g}$.

A T-joint consists of two revolute joints intersecting and perpendicular to each other,

$$
\hat{T}\left(\theta_{1}, \theta_{2}\right)=\hat{G}_{1}\left(\theta_{1}\right) \hat{G}_{2}\left(\theta_{2}\right) .
$$

The screw axes corresponding to each of the dual quaternions are defined as $\mathrm{G}_{1}=\mathbf{g}_{1}+\epsilon \mathbf{g}_{1}^{0}$ and $\mathrm{G}_{2}=\mathbf{g}_{2}+\epsilon \mathbf{g}_{2}^{0}$, so that $\mathrm{G}_{1}$. $\mathrm{G}_{2}=0$. They intersect at a point $\mathbf{c}=\left(c_{x}, c_{y}, c_{z}\right)$. Considering $\mathbf{g}_{1}^{0}=\mathbf{c} \times \mathbf{g}_{1}$ and $\mathbf{g}_{2}^{0}=\mathbf{c} \times \mathbf{g}_{2}$, the dual quaternion expression for a universal joint is

$$
\begin{array}{r}
\hat{T}\left(\theta_{1}, \theta_{2}\right)=\left\{\begin{array}{c}
\left.\mathbf{g}_{1} s_{\theta_{1}} c_{\theta_{2}}+\mathbf{g}_{2} c_{\theta_{1}} s_{\theta_{2}}+\mathbf{g}_{1} \times \mathbf{g}_{2} s_{\theta_{1}} s_{\theta_{2}}\right\} \\
c_{\theta_{1}} c_{\theta_{2}}
\end{array}\right\}+\epsilon \\
\left\{\begin{array}{c}
\mathbf{c} \times\left(\mathbf{g}_{1} s_{\theta_{1}} c_{\theta_{2}}+\mathbf{g}_{2} c_{\theta_{1}} s_{\theta_{2}}+\left(\mathbf{g}_{1} \times \mathbf{g}_{2}\right) s_{\theta_{1}} s_{\theta_{2}}\right) \\
0
\end{array}\right\},
\end{array}
$$

where $s_{\theta_{i}}=\sin \frac{\theta_{i}}{2}$ and $c_{\theta_{i}}=\cos \frac{\theta_{i}}{2}$.

An S-joint can be defined as three R-joints with concurrent axes,

$$
\hat{S}\left(\theta_{1}, \theta_{2}, \theta_{3}\right)=\hat{G}_{1}\left(\theta_{1}\right) \hat{G}_{2}\left(\theta_{2}\right) \hat{G}_{3}\left(\theta_{3}\right),
$$

with screw axes $\mathrm{G}_{1}=\mathrm{g}_{1}+\epsilon \mathbf{g}_{1}^{0}, \quad \mathrm{G}_{2}=\mathbf{g}_{2}+\epsilon \mathbf{g}_{2}^{0} \quad$ and $\mathrm{G}_{3}=\mathbf{g}_{3}+\epsilon \mathbf{g}_{3}^{0}$, perpendicular to each other and intersecting at a point $\mathbf{c}=\left(c_{x}, c_{y}, c_{z}\right)$. Incorporating these constraints into Eq. (5) we obtain

$$
\hat{S}\left(\theta_{1}, \theta_{2}, \theta_{3}\right)=\left\{\begin{array}{c}
\alpha_{1} \mathrm{G}_{1}+\alpha_{2} \mathrm{G}_{2}+\alpha_{3} \mathrm{G}_{3} \\
\alpha_{4}
\end{array}\right\},
$$

where the $\alpha_{k}$ 's appear as combinations of the joint variables $\theta_{1}, \theta_{2}$ and $\theta_{3}$,

$$
\begin{aligned}
& \alpha_{1}=\sin \frac{\theta_{1}}{2} \cos \frac{\theta_{2}}{2} \cos \frac{\theta_{3}}{2}+\cos \frac{\theta_{1}}{2} \sin \frac{\theta_{2}}{2} \sin \frac{\theta_{3}}{2}, \\
& \alpha_{2}=\cos \frac{\theta_{1}}{2} \sin \frac{\theta_{2}}{2} \cos \frac{\theta_{3}}{2}-\sin \frac{\theta_{1}}{2} \cos \frac{\theta_{2}}{2} \sin \frac{\theta_{3}}{2}, \\
& \alpha_{3}=\sin \frac{\theta_{1}}{2} \sin \frac{\theta_{2}}{2} \cos \frac{\theta_{3}}{2}+\cos \frac{\theta_{1}}{2} \cos \frac{\theta_{2}}{2} \sin \frac{\theta_{3}}{2}, \\
& \alpha_{4}=\cos \frac{\theta_{1}}{2} \cos \frac{\theta_{2}}{2} \cos \frac{\theta_{3}}{2}-\sin \frac{\theta_{1}}{2} \sin \frac{\theta_{2}}{2} \sin \frac{\theta_{3}}{2} .
\end{aligned}
$$

The design variables are the structural parameters that define the position of the joints: a point $\mathbf{c}$ on the line and the direction $\mathrm{g}$ of the line. In the case of the $\mathrm{S}$ joint, only the intersection point $\mathbf{c}$ needs to be calculated.

\section{B. Design Equations}

A total of five kinematics equations are defined: head $\left(\hat{Q}_{h e a d}\right)$; right and left arm $\left(\hat{Q}_{a r m}\right)$; and right and left leg $\left(\hat{Q}_{l e g}\right)$,

$$
\begin{aligned}
& \hat{Q}_{\text {head }}=\hat{G}_{\text {head }} \hat{S}_{\text {neck }}\left(\theta_{h 1}, \theta_{h 2}, \theta_{h 3}\right), \\
& \hat{Q}_{a r m}=\hat{G}_{\text {arm }} \hat{S}_{\text {shld }}\left(\theta_{a 1}, \theta_{a 2}, \theta_{a 3}\right) \hat{T}_{\text {elb }}\left(\theta_{a 4}, \theta_{a 5}\right) \hat{R}_{\text {wrst }}\left(\theta_{a 6}\right), \\
& \hat{Q}_{\text {leg }}=\hat{G}_{\text {leg }} \hat{S}_{\text {hip }}\left(\theta_{l 1}, \theta_{l 2}, \theta_{l 3}\right) \hat{T}_{\text {knee }}\left(\theta_{l 4}, \theta_{l 5}\right) \hat{R}_{\text {ankle }}\left(\theta_{l 6}\right) .
\end{aligned}
$$

The reference pose of the kinematic skeleton is arbitrarily chosen to coincide with the captured subject's pose at $t=0$. To create the design equations, we equate Eq. (6) to the input data, $\hat{P}^{i}$, for as many frames $i$ as are needed to solve for the design variables.

$$
\begin{aligned}
\mathcal{Q}_{\text {head }_{i}} & : \hat{Q}_{\text {head }}-\hat{P}_{\text {head }}^{i}=0, \quad i=1 \ldots n, \\
\mathcal{Q}_{\text {arm }_{i}}: & \hat{Q}_{\text {arm }}-\hat{P}_{\text {arm }}^{i}=0, \quad i=1 \ldots n, \\
\mathcal{Q}_{\text {leg }_{i}}: & \hat{Q}_{\text {leg }}-\hat{P}_{\text {leg }}^{i}=0, \quad i=1 \ldots n .
\end{aligned}
$$

For each limb, $\hat{P}^{i}$ defines the relative position of a frame attached to a point of the limb (Fig. 3 (c)), with respect to the reference kinematic skeleton.

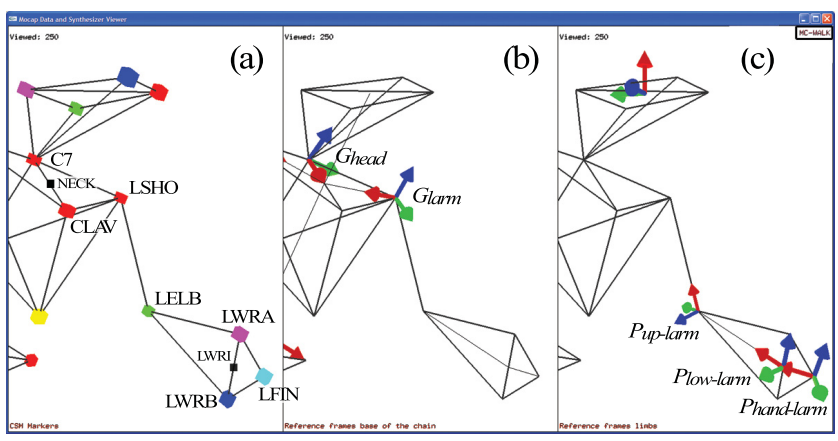

Fig. 3. Motion capture data markers (a) used to specify the reference frames $G_{\text {arm }}$ (b) and $P_{\text {larm }}:\left\{P_{\text {up-larm }}, P_{\text {low-larm }}, P_{\text {hand-larm }}\right\}$ (c) for a left arm.

As the subject moves in a three-dimensional world performing a sequence of arbitrary movements such as walking, jumping or running, the reference frames attached to the base of each chain, $G_{\text {head }}, G_{\text {arm }}$ and $G_{\text {leg }}$, are also moving (Fig. 3 (b)). Therefore, a different set of displacements $\hat{G}_{\text {head }_{i}}, \hat{G}_{\text {arm }_{i}}$ and $\hat{G}_{\operatorname{leg}_{i}}$ for at each $t_{i}$ exists. Expressing them with respect to the reference configuration pose at $t_{0}$, we obtain

$$
\hat{G}_{\text {chain }}^{i}=\hat{G}_{\text {chain }_{i}} \hat{G}_{\text {chain }}^{*}, \quad i=1 \ldots n,
$$

where the subscript chain is used to denote any of the five chains that form the skeleton: head, left and right arm, and left and right leg. Once the equations are stated and the task positions are calculated, we solve individually for each of the five chains.

\section{Hierarchical Synthesis}

In the hierarchical synthesis (Fig. 4), we solve sequentially link by link along the chain, starting the procedure at the link closest to the base of the chain. In the case of an arm, the process starts at the upper $\operatorname{arm}\left(\hat{P}_{u p}^{i}\right)$ to solve for the shoulder 
joint $\left(\hat{S}_{\text {shld }}\left(\theta_{a 1}^{i}, \theta_{a 2}^{i}, \theta_{a 3}^{i}\right)\right)$, continues with the lower arm $\left(\hat{P}_{\text {low }}^{i}\right)$ determining the elbow joint $\left(\hat{T}_{e l b}\left(\theta_{a 4}^{i}, \theta_{a 5}^{i}\right)\right)$, and finishes with the hand $\left(\hat{P}_{\text {hand }}^{i}\right)$ and wrist joint $\left(\hat{R}_{\text {wrst }}\left(\theta_{a 6}^{i}\right)\right)$.

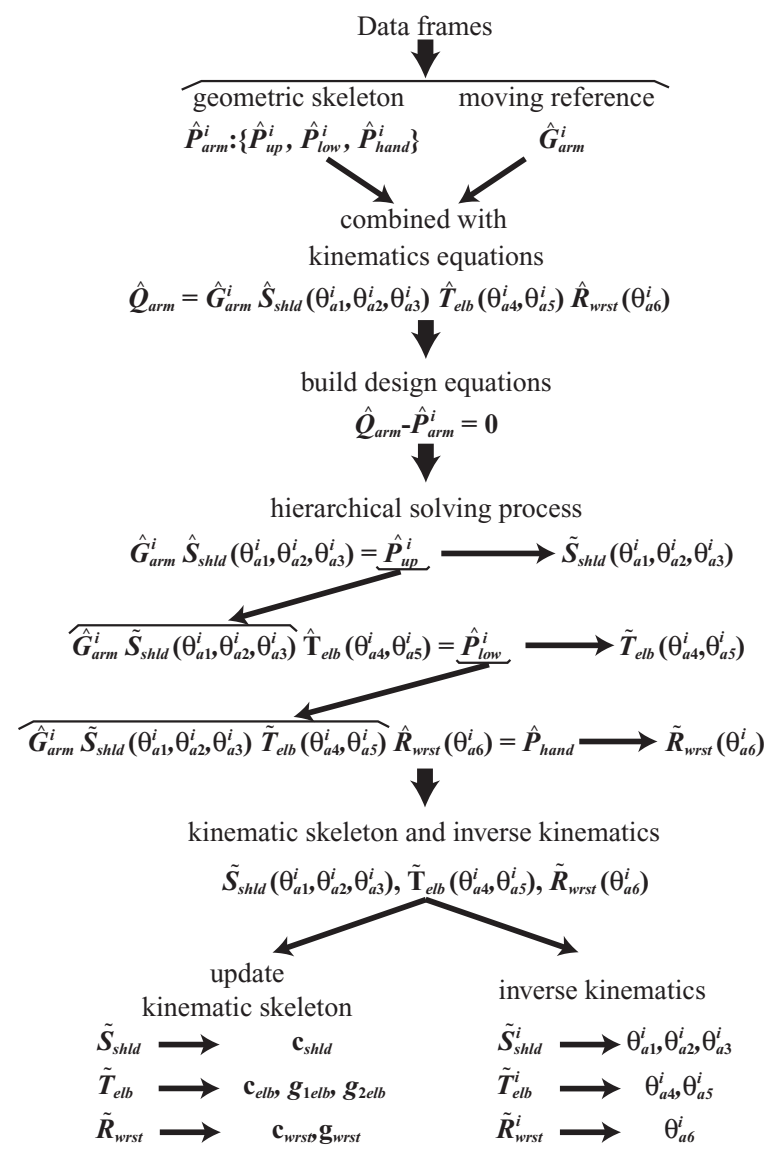

Fig. 4. Hierarchical synthesis of an arm at $t_{i}$.

Taking an arm as an example, we need to solve for

$$
\mathcal{Q}_{a r m}: \hat{Q}_{a r m}-\hat{P}_{a r m}^{i}=0, \quad i=1 \ldots n,
$$

The number of data frames $n$ required to obtain a solution depends on the type of joint being solved and the number of unknowns.

The hierarchical solution process begins at the S-joint defining the shoulder of the $\hat{Q}_{a r m}$ chain, and the set of equations,

$$
\hat{G}_{a r m}^{i} \hat{S}_{\text {shld }}\left(\theta_{a 1}^{i}, \theta_{a 2}^{i}, \theta_{a 3}^{i}\right)=\hat{P}_{u p}^{i}, \quad i=1 \ldots n,
$$

which is equivalent to

$$
\hat{S}_{\text {shld }}\left(\theta_{a 1}^{i}, \theta_{a 2}^{i}, \theta_{a 3}^{i}\right)=\hat{G}_{a r m}^{i *} \hat{P}_{u p}^{i}, \quad i=1 \ldots n,
$$

and solved numerically to obtain the point defining the location of the S-joint, $\mathbf{c}$, and the joint angles $\theta_{a 1}^{i}, \theta_{a 2}^{i}, \theta_{a 3}^{i}$ that most closely approximate the position at $t_{i}$. This allows us for the creation to create the dual quaternion $\tilde{S}_{\text {shld }}\left(\theta_{a 1}^{i}, \theta_{a 2}^{i}, \theta_{a 3}^{i}\right)$ approximating the movement of the upper arm.

To solve for the elbow, the input data of the lower arm must be equated with the series of transformations from the base to the lower arm,

$$
\hat{G}_{\text {arm }}^{i} \hat{S}_{\text {shld }}\left(\theta_{a 1}^{i}, \theta_{a 2}^{i}, \theta_{a 3}^{i}\right) \hat{T}_{e l b}\left(\theta_{a 4}^{i}, \theta_{a 5}^{i}\right)=\hat{P}_{l o w}^{i}, i=1 \ldots n .
$$

To isolate the elbow joint, we left-multiply by the inverse of $\hat{G}_{a r m}^{i} \tilde{S}_{\text {shld }}\left(\theta_{a 1}^{i}, \theta_{a 2}^{i}, \theta_{a 3}^{i}\right)$, which is the same as $\hat{P}_{u p}^{i}$ (Eq. (8)). This is done using the dual quaternion conjugate,

$$
\hat{T}_{e l b}\left(\theta_{a 4}^{i}, \theta_{a 5}^{i}\right)=\hat{P}_{u p}^{i^{*}} \hat{P}_{l o w}^{i}, \quad i=1 \ldots n,
$$

for which the right part of the equation is known. After solving numerically, we obtain the Plücker coordinates of the joint axis and the joint rotation, which can be used to create the dual quaternion approximating the elbow joint $\tilde{T}_{e l b}\left(\theta_{a 4}^{i}, \theta_{a 5}^{i}\right)$.

Similarly to the solving process used for the elbow, we use data from the movement of the hand to solve for the last joint of the chain

$$
\begin{array}{r}
\hat{G}_{a r m}^{i} \hat{S}_{\text {shld }}\left(\theta_{a 1}^{i}, \theta_{a 2}^{i}, \theta_{a 3}^{i}\right) \hat{T}_{\text {elb }}\left(\theta_{a 4}^{i}, \theta_{a 5}^{i}\right) \hat{R}_{w r s t}\left(\theta_{a 6}^{i}\right)=\hat{P}_{\text {hand }}^{i}, \\
i=1 \ldots n .
\end{array}
$$

After pre-multiplying by the lower limb data, the design equations for the wrist are

$$
\hat{R}_{w r s t}\left(\theta_{a 6}^{i}\right)=P_{\text {low }}^{i^{*}} \hat{P}_{\text {hand }}^{i}, \quad i=1 \ldots n,
$$

providing the approximation $\tilde{R}_{w r s t}\left(\theta_{a 6}^{i}\right)$ for the wrist.

To obtain an accurate kinematic skeleton the input movement has to be general enough to show movement at all joints. This process is repeated several times, accounting for the lack of resolution of the input data, and for the disparity between a model made of rigid rotations and the real movement of the human body. The synthesis is performed for a number of steps, averaging the solutions at each joint, until the error between solutions satisfies an error threshold.

\section{Minimization and Measuring}

For the experimental results, a Levenberg-Marquardt algorithm [18] was used to solve the design equations (Eq. (7)). The algorithm showed robustness and a fast convergence rate to the correct solution for the five chains modeling the human subject, allowing for interactive rates of the kinematic synthesis process.

1) Error Measurement: Once a solution is obtained, the error at each joint for an instant in time $i$ is defined as the twonorm error $\epsilon^{i}$ of the 8-component dual quaternion difference obtained from subtracting the dual quaternion data from the solution. For an arm $\epsilon^{i}$ is defined as,

$$
\epsilon^{i}=\left\|\tilde{Q}_{a r m}^{i}-\hat{P}_{a r m}^{i}\right\|_{2}=\sqrt{\sum_{j=1}^{8}\left(\tilde{Q}_{a r m}^{i}-\hat{P}_{a r m}^{i}\right)_{j}^{2}},
$$

which is equivalent to computing the length of the difference vector $\left(\tilde{Q}_{a r m}^{i}-\hat{P}_{a r m}^{i}\right)$. This metric for spatial displacements, as any other metric that we can define, is not bi-invariant [19]. More research is required in order to assess the effects of wandering subjects on the metrics. 
2) Execution Time Measurement: Execution time quantifies the current computational cost required by the synthesis process. To measure the execution time, a timer is initiated when the synthesis process starts for a specific joint, and it is stopped once it reaches a valid solution; i.e. the error value $\epsilon^{i}$ is below a predefined threshold.

\section{RESUlts}

Ten case studies were evaluated quantitatively and qualitatively for synthetic and real data records. Table I presents their characteristics, including a reference number, dataset name, input data source type (synthetic or motion capture for real human subjects), total length in frames of the sequence, and acquisition rate in frames per second (fps) for the motion capture sequences. Mathematica [20] was used to generate the synthetic datasets. Experiments were run on a Dell Inspiron 8600 laptop with a $1.4 \mathrm{GHz}$ Pentium M processor, 768 MB of RAM, and nVidia Geforce FX Go5200 graphics.

TABLE I

CHARACTERISTICS OF ANALYZED CASE STUDIES [14].

\begin{tabular}{c|l|l|c|c}
\hline Nr & Dataset Name & Source Type & Length & Rate \\
\hline \hline 1 & MATH-STR & Synthetic & 40 & - \\
2 & MATH-NOISY-STR & Synthetic & 40 & - \\
3 & MC-ARMS & Motion capture & 1545 & 120 \\
4 & MC-LEGS & Motion capture & 1476 & 120 \\
5 & MC-WALK & Motion capture & 1491 & 120 \\
6 & MC-WANDER & Motion capture & 1505 & 120 \\
7 & MALE-WALKA & Motion capture & 331 & 60 \\
8 & MALE-WALKB & Motion capture & 669 & 60 \\
9 & FEMALE-WALK & Motion capture & 962 & 60 \\
10 & BALLET & Motion capture & 464 & 30 \\
\hline
\end{tabular}

MATH-STR tests the accuracy and correctness of the algorithm, given an ideal dataset, and MATH-NOISY-STR evaluates its robustness, after introducing random noise at the joint locations. The remaining datasets correspond to motion performed by real subjects. Datasets three to six captured a single subject using an optical motion capture system available in the Dance Department at the University of California, Irvine, while datasets seven to ten were available for public download.

Performance is quantified examining synthesis errors and execution times (Sect. IV-D), while the recovered sequences are qualitatively validated through visual inspection. First, one case study is discussed in detail and finally, a comprehensive comparison for all cases is included to evaluate the global performance of the proposed method.

\section{A. Dataset MC-WANDER}

In MC-WANDER a woman walks moving arms, legs and head. For the synthesis, the original sequence was subsampled, resulting in an effective length of 131 frames and 12 fps. Fig. 5 shows a sequence of inputs and results, where rows correspond to frame numbers 1250 and 1290. The first column shows the input motion capture data used for the motion synthesis process, the second the recovered motion, while the third one compares them blending both views. The last column contains the kinematic skeleton at the reference configuration, which should remain constant during the whole sequence.

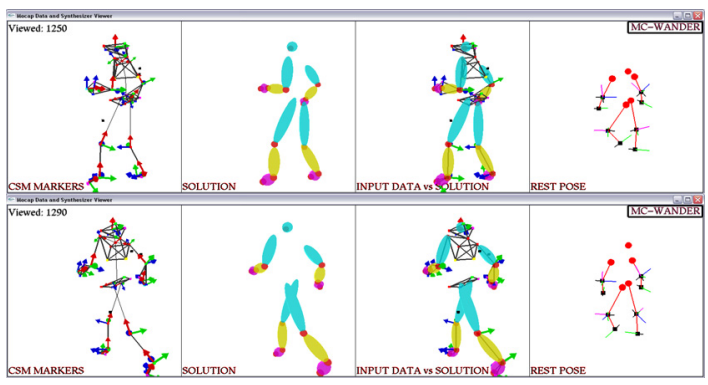

Fig. 5. MC-WANDER legs synthesis comparison (frames 1250 and 1290).

Since sufficient movement is available for all body parts, visual results of the synthesized sequence are stable for all chains. Table II presents the mean values and standard deviations for the structural design parameters characterizing a left arm in the kinematic skeleton, respectively using 93, 117 and 86 samples for shoulder, elbow and wrist. As the analyzed motion is generic, execution times on average are constant for all chains and accordingly for the global average of the subject. As a general trend T-joints synthesis is computationally more demanding, which compared to S- and R-joints is consistent, given the larger number of parameters to synthesize.

TABLE II

KINEMATIC SKELETON FOR A LEFT ARM IN MC-WANDER.

\begin{tabular}{l|c|c}
\hline Joint & Params & Mean and Standard Dev \\
\hline \hline Shoulder & $\mathrm{c}$ & $(0.3233,0.3554,1.1951) \pm(0.0570,0.0176,0.0429)$ \\
\hline Elbow & $\mathrm{c}$ & $(0.3937,0.2941,0.9308) \pm(0.0341,0.0155,0.0078)$ \\
& $\mathrm{g} 1$ & $(0.9296,0.1596,0.3320) 17.74^{\circ} \pm 11.33^{\circ}$ \\
& $\mathrm{g} 2$ & $(-0.3597,0.5878,0.7245) 17.41^{\circ} \pm 10.99^{\circ}$ \\
\hline Wrist & $\mathrm{c}$ & $(0.4001,0.1807,0.7616) \pm(0.0181,0.0264,0.0253)$ \\
& $\mathrm{g}$ & $(-0.0299,0.8988,-0.4372) 26.03^{\circ} \pm 12.64^{\circ}$ \\
\hline
\end{tabular}

\section{B. Case Studies Comparative}

Fig. 6 compares error (left) and execution times (right) for each chain (head, arms and legs) averaging all case studies, while Fig. 7 averages all joints for each case study. In Fig. 6, all chains are in the same error and execution time range. However, only those cases presenting a generic motion at all joints show such behavior with respect to error. In terms of execution times, except for MC-ARMS, all case studies are on average in the 1.263-6.353 milliseconds range.

Based on the joint types (Fig. 7), a repetitive pattern is found in all case studies, $\mathrm{S}$-joints synthesis error is at least twice the error for R- or T-joints. R-and T-joints do not present a clear pattern, but are comparable in terms of error. The use of S-joints to approximate neck, shoulders and hips are not that accurate, requiring their topological redesign. Shoulders show a three-degrees-of-freedom movement, but assuming 
three concurrent axis is excessive. Execution times for T-joints are significantly larger compared to $\mathrm{S}$ - and R-joints, being consistent with the larger number of synthesis parameters for a T-joint. Nevertheless, T-joints present a low synthesis error, indicating a suitable approximation.
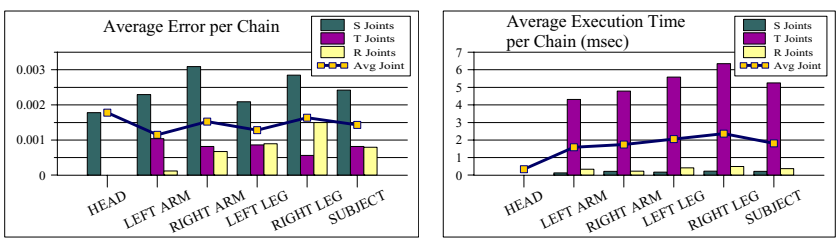

Fig. 6. Per chain average error (left) and execution time (right) comparison.

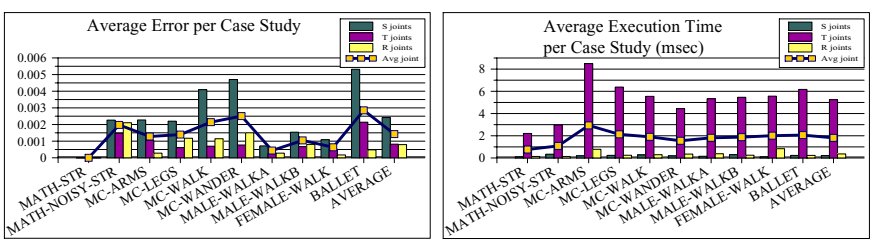

Fig. 7. Global comparison of average error (left) and execution time (right).

Real datasets required at most four input data frames to provide a first acceptable approximation, in contrast to the 12 or 15 needed for the synthetic ones. The fast convergence rate for real data sequences underlines the richness of real human motion. Assuming all joints were synthesized using the worstcase execution time for each joint type, the average synthesis frame rate would be in the range of $1.98 \mathrm{fps}$. However, on average this frame rate increases to $42.44 \mathrm{fps}$, which allows for interactive synthesis rates. The average execution times for the experimental results confirm that synthesis is feasible at interactive rates. In addition, frames causing longer execution times can be directly correlated with those that show poor qualitative synthesis performance. Therefore, they can be discarded by filtering out time-consuming data frames.

\section{CONCLUSIONS}

A technique is presented for the simulation of human-like motion applied to the design of humanoid robots or 3D avatars. With finite-position kinematic synthesis using dual quaternions, a kinematic skeleton is synthesized to approximate the set of poses specified for an arbitrary subject. The advantage is that complicated articulated figures and joint types, accepting arbitrary poses of human subjects, can be created without human intervention. Experiments using real optical motion capture data show that the algorithm is fast, stable and reliable.

The human skeleton is modeled as four S-T-R chains for the limbs and one $\mathrm{S}$ chain for the head. Although this model could be extended to reflect more complex topologies, the results show that the error between real motion and the kinematic skeleton approximation can be small even when using simple kinematic structures.
On average, all chains used in the experiments were within the same synthesis error range, indicating it can be bounded. Average execution times are stable, and show that synthesis can be performed at interactive rates.

The results obtained can be applied to create libraries of human motion and 3D figures for motion retargetting applications, as well as to provide valuable information about the topologies that accurately imitate human motion.

\section{ACKNOWLEDGMENTS}

This research was supported in part by the CaliforniaCatalonia Engineering Innovation Program.

\section{REFERENCES}

[1] J. O’Brien, R. Bodenheimer, G. Brostow, and J. Hodgins, "Automatic joint parameter estimation from magnetic motion capture data," in Graphics Interface, May 2000, pp. 53-60.

[2] C. Bregler and J. Malik, "Tracking people with twists and exponential maps," in IEEE Conference on Computer Vision and Pattern Recognition, June 1998, pp. 8-15.

[3] I. Mikić, M. Triverdi, E. Hunter, and P. Cosman, "Articulated body posture estimation from multi-camera voxel data," in IEEE Conference on Computer Vision and Pattern Recognition, vol. I, December 2001, pp. $455-460$.

[4] C.-W. Chu, O. C. Jenkins, and M. J. Matarić, "Markerless kinematic model and motion capture from volume sequences," in Computer Vision and Pattern Recognition, vol. II, June 2003, pp. 475-482.

[5] T. Beth, I. Boesnach, M. Haimerl, J. Moldenhauer, K. Bös, and V. Wank, "Characteristics in human motion - from acquisition to analysis," in IEEE International Conference on Humanoid Robots, Karlsruhe, 2003, p. 56.

[6] J. Moldenhauer, I. Boesnach, T. Beth, V. Wank, and K. Bös, "Analysis of human motion for humanoid robots," in IEEE International Conference on Robotics and Automation, 2005, pp. 312-317.

[7] J. Lee and S. Y. Shin, "A hierarchical approach to interactive motion editing for human-like figures," in ACM SIGGRAPH, 1999, pp. 39-48.

[8] M. Brand and A. Hertzmann, "Style machines," in ACM SIGGRAPH, 2000, pp. 183-192.

[9] M. Abe, T. Yamamoto, and T. Fujinami, "A dynamical analysis of kneading using a motion capture device," in proceedings of third international workshop on Epigenetic Robotics, 2003, pp. 41-48.

[10] E. Lee and C. Mavroidis, "Geometric design of 3R manipulators for reaching four end-effector spatial poses," The International Journal of Robotics Research, vol. 23, no. 3, pp. 247-254, 2004.

[11] A. Perez and J. M. McCarthy, "Dual quaternion synthesis of constrained robotic systems," ASME Journal of Mechanical Design, vol. 126, no. 3 , pp. 425-435, 2004.

[12] G. Mullineux, "Modeling spatial displacements using Clifford algebra," ASME Journal of Mechanical Design, vol. 124, no. 3, pp. 420-424, 2004.

[13] E. Bayro-Corrochano, K. Daniilidis, and G. Sommer, "Motor algebra for 3D kinematics. the case of the hand-eye calibration," International Journal of Mathematical Imaging and Vision, vol. 13, no. 2, pp. 79-99, 2000.

[14] M. Villa-Uriol, "Video-based avatar reconstruction and motion capture," Ph.D. dissertation, Henry Samueli School of Engineering, University of California, Irvine, 325 Engineering Tower, Irvine, CA, 2005.

[15] J. J. Craig, Introduction to Robotics, Mechanics and Control. Addison Wesley Publ. Co, 1989.

[16] J. M. McCarthy, Introduction to Theoretical Kinematics. Cambridge, MA: The MIT Press, 1990.

[17] J. M. Selig, Geometrical Methods in Robotics. Springer-Verlag, New York, 1996.

[18] J. J. Moré, B. S. Garbow, and K. E. Hillstrom, "User guide for MINPACK-1," Argonne National Laboratory, Tech. Rep. ANL-80-74, 1980.

[19] J. Martinez and J. Duffy, "On the metrics of rigid body displacements for infinite and finite bodies," ASME Journal of Mechanical Design, vol. 117, pp. 41-47, 1995

[20] Wolfram Research Inc., "http://www.wolfram.com/," July 2005. 